\title{
Integrating Biochemical Separation and Purification Steps in Fermentation Processes
}

\author{
HENRY Y. WANG \\ Department of Chemical Engineering \\ The University of Michigan \\ Ann Arbor, Michigan 48109
}

\section{INTRODUCTION}

Owing to recent developments in molecular biology and biotechnology, we have seen rapid growth in the development of industrial processes to produce valuable substances by microbial means. The idea is not new to the fermentation technologist because they are the means for producing beverages, antibiotics, and many other complex organic materials for commercial use. There are some inherent engineering disadvantages of fermentation processes as compared to conventional chemical processes that need to be solved in order to make these processes economically attractive. The traditional fermentation process involves growing a pure culture of a microorganism that produces the product of interest in a fermenter after sterilization and inoculation. The fermentation can be carried out in batch, semicontinuous, and continuous modes. Depending on whether the desired product is intracellular or extracellular, the harvested broth is then subjected to various separation and purification steps until the specific product of interest is isolated from the fermentation broth. The common steps involved are cell removal, volume reduction, isolation, and purification until the specified product purity has been achieved.' The fermentation and the subsequent recovery steps have traditionally been carried out in separate departments and interaction among these groups has been minimal. Kaufman and $\mathrm{Paul}^{2}$ pointed out the interactive nature of these steps, especially between the fermentation and its subsequent product recovery scheme. In this paper, this interactive nature can be examined and utilized to optimize the entire biochemical process. A hybrid extractive fermentation system developed at the University of Michigan will be used as a model system to illustrate this point.

\section{PROBLEMS ASSOCIATED WITH FERMENTATION AND RECOVERY}

The microbial fermentation process has become an integral part of the recent advances in genetic engineering and biotechnology. Most of the fermentation processes are complex processes that involve multiple substrate, gas-liquid mass transfer, and sometimes non-Newtonian broth rheology. The final desired product concentration in the aqueous medium can vary over a wide range. Some of the organic acid fermentations such as citric acid and glutamic acid, a concentration of 100 grams per liter or above can be achieved. On the other hand, some more complex macromolecular product fermentations, such as vitamin $B_{12}$ and human insulin, a concentration of 1 
gram per liter or less is satisfactory because these products command premium prices. As shown in FigurE 1, the fermentation product cost is more or less dictated by the final product concentration in the fermentation broth. It is always desirable to increase the final product concentration in the fermentation broth. The primary causes of the dilute nature of the fermentation end product are that the product itself can stop its own synthesis (product regulation) and product degradation in the fermenting broth. Even though these effects that occur at high product concentration are known, approaches for major improvement have been primarily limited to genetic manipulation. By means of random mutation and selection, strains tolerant to a high concentration of product may be selected to avoid such problems.

Isolation of microbial products produced by fermentation is a specialized field because of the unique character of the fermentation broth from which the products are synthesized. The complexity of the heterogenous mixture of soluble and insoluble materials is required to maintain or improve the fermentation, but it also causes problems for the separation scientists and engineers. Although not always, the product usually is present in a relatively low concentration compared to the concentration of other materials present. The desired product is also prone to degradation by extremes of temperature and $\mathrm{pH}$. Most important of all, the product must be protected from both enzymatic/microbial and chemical degradations. Depending on whether the fermentation product is intracellular or extracellular, the sequence of recovery and purification steps is shown in FiGURE 2 . The extraction efficiency of a product recovery and purification scheme is dictated not only by the final fermentation product concentration but also by the number of steps required to achieve the specified product purity that, in turn, is dictated by the market demand. Increasing the number of steps in the isolation of microbial product from the fermentation broth will increase the

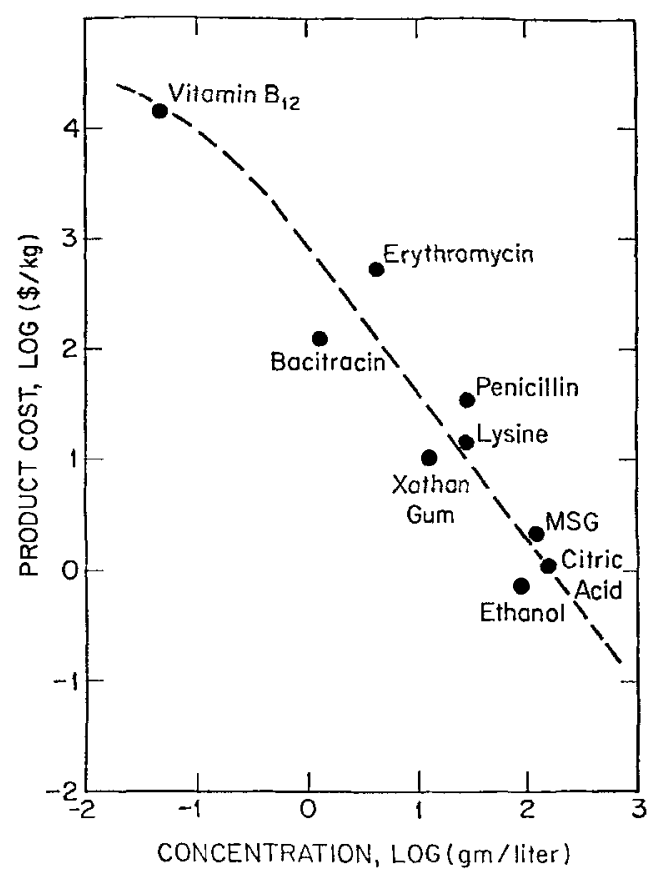

FIGURE 1. Sales price of various fermentation products versus the final fermentation product concentrations. 
FIGURE 2. Various steps involved in fermentation processes and subsequent recovery processes.

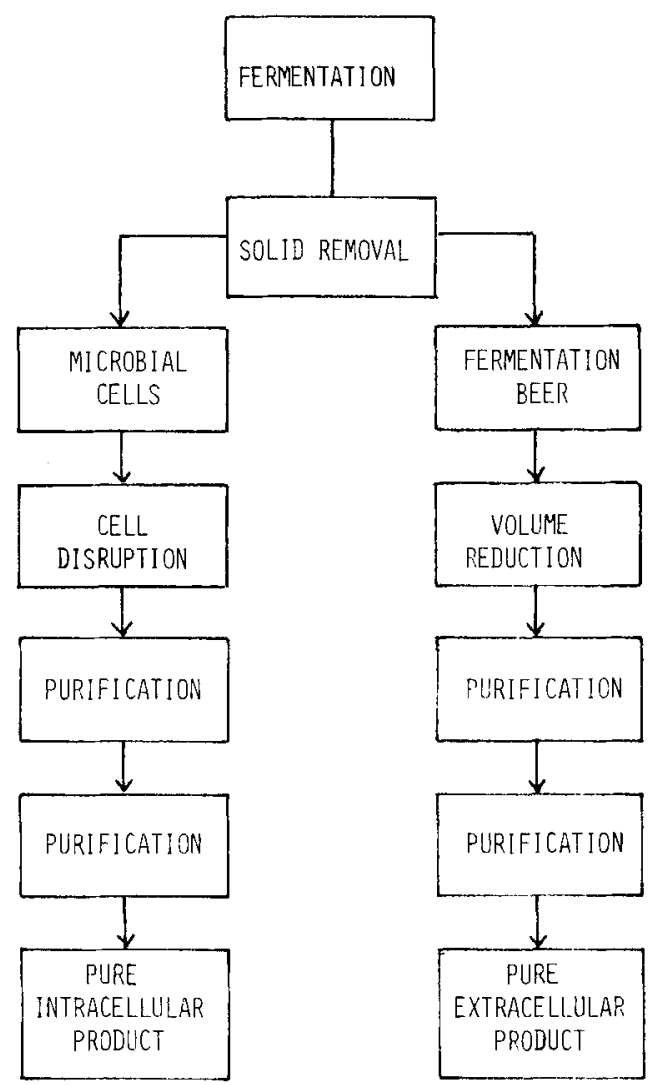

product purity, but it also decreases the overall extraction efficiency (FIG. 3). The initial isolation steps such as cell removal and volume reduction generally cause a more drastic drop in the overall extraction efficiency as depicted in FIGURE 3.

\section{HYBRID EXTRACTION-FERMENTATION SYSTEM}

The need to achieve high product concentration in the fermentation broth is essential for both the fermentation (product yield) and recovery (extraction efficiency). One engineering approach is to couple the extraction with the fermentation step so that the inhibitory metabolic products can be continuously removed from the aqueous broth so that the inhibitory effects and/or degradation phenomena can be avoided or minimized throughout the fermentation. In this manner, the product formed may also be concentrated, which will facilitate the eventual purification steps as well as avoiding the metabolic inhibition and degradation phenomena. Various attempts have been made to improve fermentation yield and alleviate product inhibition using ion-exchange resins, solvents, and membrane processes (TABLE 1). Certain criteria of the specific extractant must be met in order to carry out this type of 


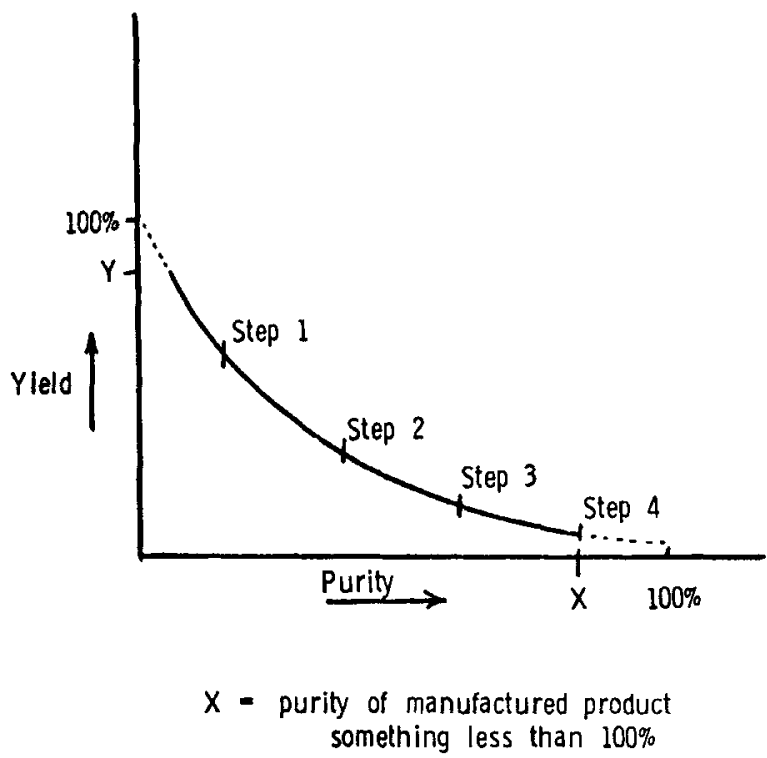

FIGURE 3. Yield-Purity relationship. The extraction efficiency as a function of product purity.

extraction-fermentation process (TABLE 2). After reviewing various separating agents, such as nonaqueous solvents, ion-exchange resins, and activated carbon, we found that the nonionic polymeric resins such as XAD-2 and XAD-4 (Rohm and Haas, Philadelphia, PA) that do not contain any specific function groups are most suitable for this purpose.

TABLE 1. Fermentation with On-line Extraction

\begin{tabular}{|c|c|c|c|c|}
\hline Fermentation & $\begin{array}{l}\text { Separation } \\
\text { Techniques }\end{array}$ & Advantages & Disadvantages & Reference \\
\hline Antibiotics & Ion-exchange resins & Eliminate filtration & Excess ions & 3 \\
\hline Salicyclic acid & Dialysis & $\begin{array}{l}\text { Alleviate product } \\
\text { inhibition }\end{array}$ & Increased volume & 4 \\
\hline Salicyclic acid & Ion-exchange resins & $\begin{array}{l}\text { Alleviate product } \\
\text { inhibition }\end{array}$ & Excess ions & 5 \\
\hline Cycloheximide & Dialysis solvent & $\begin{array}{l}\text { Alleviate product } \\
\text { inhibition }\end{array}$ & Solvent toxicity & 6 \\
\hline Carotenoids & Kerosene grease & $\begin{array}{l}\text { Increase product } \\
\text { yield }\end{array}$ & Limited application & 8 \\
\hline Ethanol & Vacuum distillation & Increase productivity & Limited application & 8 \\
\hline Ethanol & Activated carbon & Increase productivity & Toxicity & 9 \\
\hline $\begin{array}{l}\text { Anacrobic diges- } \\
\text { tion }\end{array}$ & Activited carbon & Process stability & Recovery & 10 \\
\hline $\begin{array}{l}\text { Anaerobic diges- } \\
\text { tion }\end{array}$ & Membrane & Increase productivity & Membrane fouling & 11 \\
\hline
\end{tabular}


TABLE 2. Criteria for Extractants Used in On-line Extraction-Fermentations

1. The extraction must be selective.

2. The extractant must be nontoxic to the growing culture.

3. The extraction system must be capable of aseptic operations.

4. The extractant must have a high loading capacity for the product.

5. The extractant must be reusable if it is expensive.

\section{IMMOBILIZED PRODUCT FERMENTATION (IPF)}

Cycloheximide fermentation, antifungal and antibiotic, has been used in our investigation. Biochemical and fermentation studies of the cycloheximide fermentation have been extensively investigated. ${ }^{6,12}$ The time course for the conventional cycloheximide fermentation has been observed to be quite reproducible. The cycloheximide concentration in the fermentation broth reached a peak at $\log 168$ (Frg. 4). This

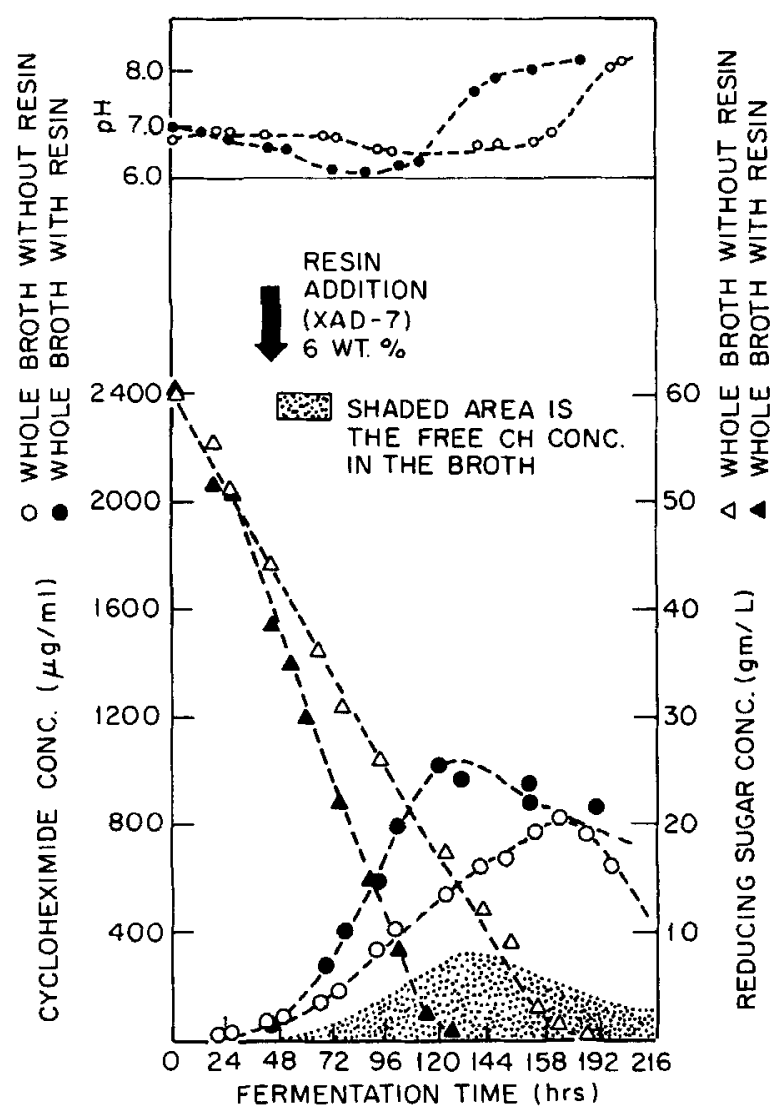

FIGURE 4. Comparison between a normal cycloheximide fermentation using 6 wt \% XAD-7 resins. The adsorbents were added at $\log 48$. 
corresponds to the depletion of glucose in the medium. The medium $\mathrm{pH}$ rose above 7.0 and product degradation occurred (cycloheximide is less stable under alkaline conditions). The maximum cycloheximide titer that we can achieve in the laboratory fermenter is about $850 \mu \mathrm{g} / \mathrm{ml}$. Kominek ${ }^{12}$ has shown this fermentation to be feedback regulated.

By adding sterilized polymeric resins such as XAD-7 and XAD-4 directly into the fermentation broth, the profiles for both glucose utilization and cycloheximide production were changed. When $6 \mathrm{wt} \%$ of XAD-7 were added at the $48 \mathrm{th}$ hour of a cycloheximide fermentation (FIG. 4), the glucose utilization rate was increased from $0.36 \mathrm{~g} / 1-\mathrm{hr}$ to $0.5 \mathrm{~g} / 1-\mathrm{hr}$ indicating that the presence of these solid adsorbents somehow change the physiological activities of the microbial culture. Since the addition was made around the 48th hour when the cell growth was completed, this enhanced glucose utilization was not accompanied with additional cell growth. The peak product yield was higher with a value of $27.8 \mathrm{mg} \mathrm{CH} / \mathrm{g}$ of glucose instead of the normal fermentation yield of $18.6 \mathrm{mg} / \mathrm{g}$ of glucose. Obviously, the adsorption rate by the XAD-7 resins was not fast enough, thus accumulation of free cycloheximide in the broth was observed (FIG. 4).

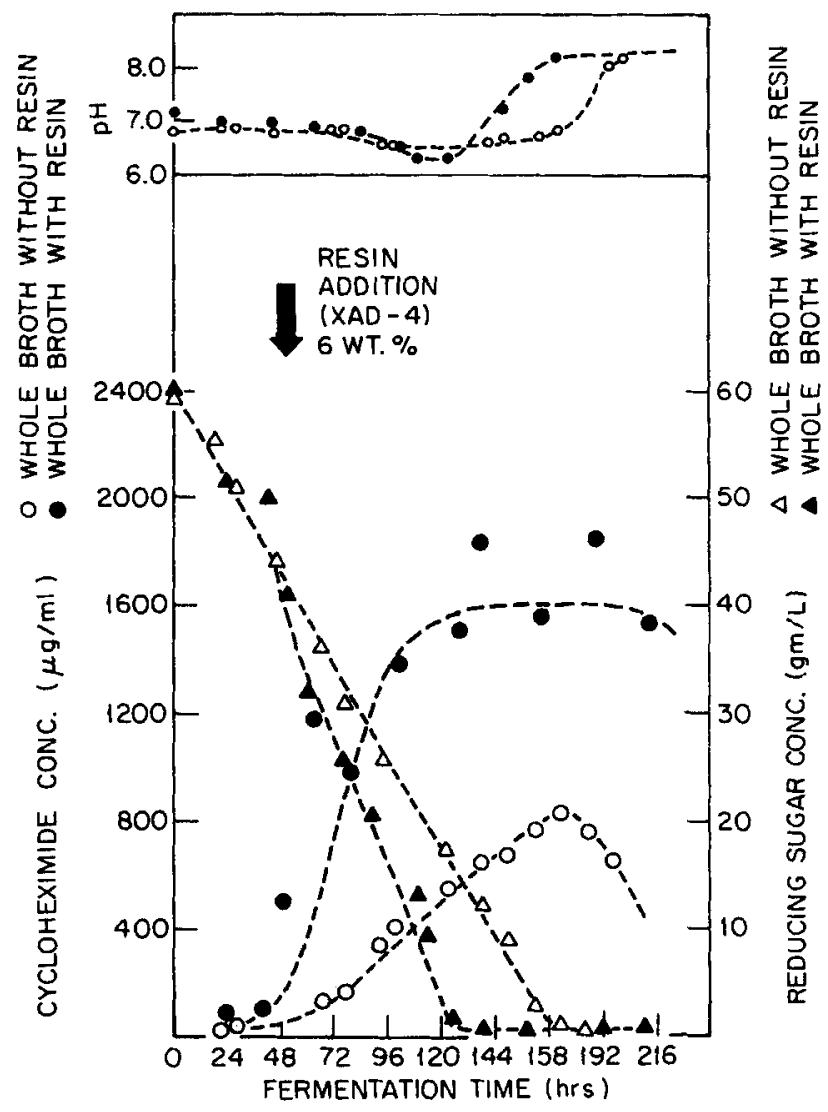

FIGURE 5. Comparison between a normal cycloheximide fermentation and resin addition cycloheximide fermentation using $6 \mathrm{wt} \%$ XAD-4 resin. The adsorbents were added at $\log 48$. 


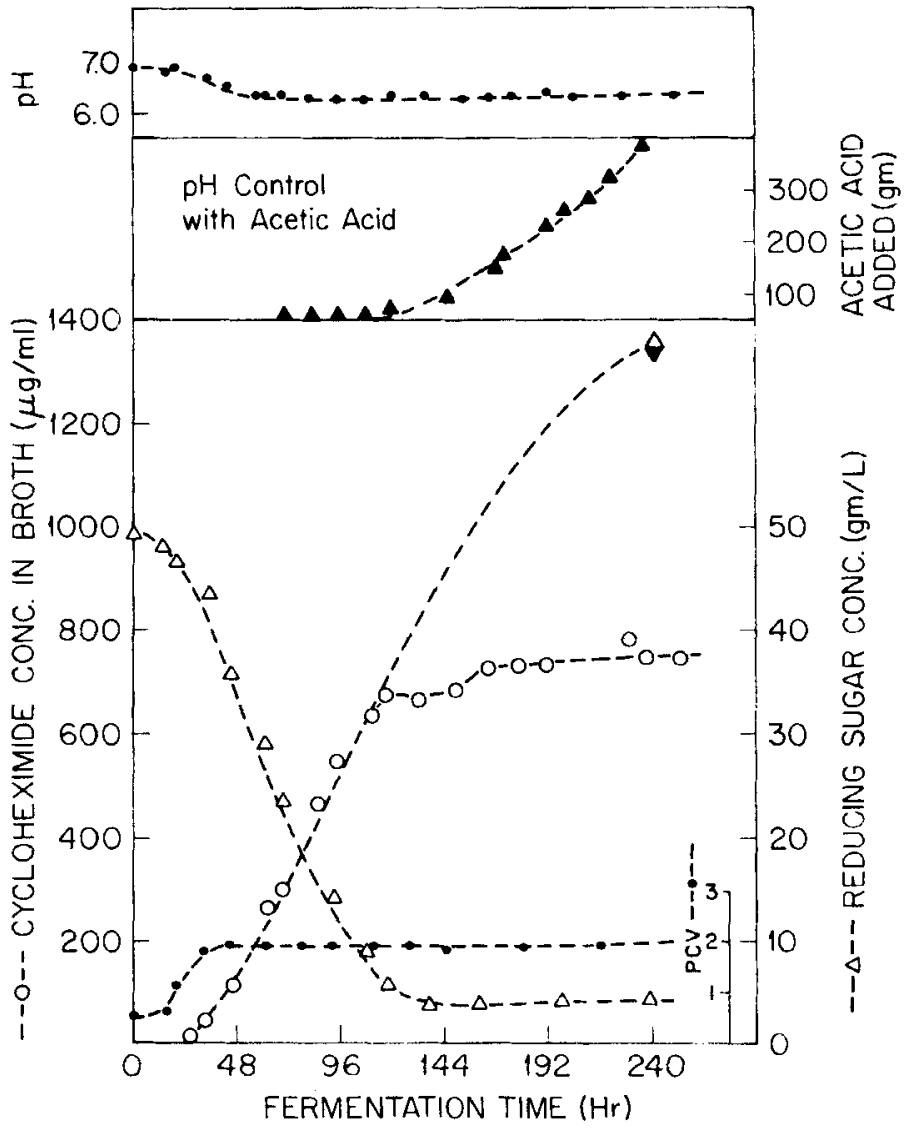

FIGURE 6. Cycloheximide accumulation in broth of the immobilized-resin fermentation.

When we used the same amount of XAD-4 resin instead of XAD-7 resin (FIG. 5), a similar phenomenon was observed. Since XAD-4 polystyrene- based polymeric resin is more hydrophobic than XAD-7 and contains more surface area, all cycloheximide produced was adsorbed onto the resins instantly and the free broth activity of cycloheximide was low throughout the fermentation period. The maximum cycloheximide production rate was $17.5 \mathrm{mg} / 1$-hr with a product yield of $36.5 \mathrm{mg} \mathrm{CH} / \mathrm{g}$ of glucose. These were substantially higher than the control fermentation run. Over $98 \%$ of the produced cycloheximide adsorbed onto the resin particles. We could conceivably harvest the antibiotic-loaded resins and drastically reduce the volume of materials that need to be processed for further separation and purification.

\section{RECOVERY OF THE POLYMERIC RESINS}

Dispersing the resins in the culture broth has several engineering problems such as clogging the valves and attrition of the resin particles which are also abrasive to the 
tank wall and the shaft seals. We have been experimenting with keeping the resins in dialysis membrane bags (Spectrapor M.W. cut off 8,000 ) that were mounted on the baffles inside the fermenter. The resin and the culture medium were sterilized together. Dense resin cakes were formed in these bags during the fermentation and diffusion into the cakes appeared to significantly limit the removal rate, and as a result cycloheximide accumulated in the culture broth (FIG. 6). Higher final cycloheximide titer was achieved, but a significant amount remained in the fermentation broth. Visual inspection of the resins in the membrane bag indicates very limited penetration by the fermenting broth. An alternative to this approach is to physically separate the resin from the culture medium in an external extractor. Broth can then be pumped between the fermenter and extractor during fermentation.

The antibiotic-loaded polymeric resins can easily be separated from the harvested culture broth using a vibrating screen or similar solid-liquid separation devices. For our studies, butyl acetate was used to elute the product from the resin. The purity of the butyl-acetate-eluted cycloheximide was then compared (TABLE 3). The cycloheximide eluted from the resins was comparable to those extracted directly from the culture broth. On the other hand, the product eluted from the resins that were enclosed in a dialysis membrane bag was considerably more pure (TABLE 3 ). These polymeric adsorbents can be reused after cleaning with caustic and methanol. Ideally, we would like to develop a system that would selectively adsorb the desired product from the fermentation broth. This can reduce subsequent purification steps. Currently, we are studying this possibility.

TABLE 3. Purity of Extracted Cycloheximide from the XAD-4 Resin

\begin{tabular}{llc}
\hline \multicolumn{1}{c}{ Fermentation } & \multicolumn{1}{c}{ Solvent } & Purity (\%) \\
\hline Shake flask (control) & Butyl acetate & 39 \\
Fermenter (control) & Butyl acetate & 24 \\
XAD-4 Resin (dispersed) & Butyl acetate & 54 \\
XAD-4 Resin in dialysis bag & Butyl acetate & 77 \\
XAD-4 Resin in dialysis bag & Methylene chloride & 74 \\
XAD-4 Resin in dialysis bag & Methanol & 42 \\
\hline
\end{tabular}

\section{CONCLUSION}

The need to increase the final product concentration in fermentation processes leads us to develop this immobilized product fermentation (IPF). By directly adding a separating agent such as XAD-4 resin that is nontoxic to the microbial cells into the fermentation broth, the antibiotic synthesis rate has been shown to increase drastically and the final product yield increases by a factor of 3 or more. The antibioticconcentrated resins can easily be separated from the fermentation broth and the antibiotic can be extracted using solvents. Volume reduction and partial purification were being demonstrated in the case of cycloheximide fermentation. Currently, we are looking into the possibility of extending this antibiotic synthesis and optimizing this IPF process through computer control.

\section{ACKNOWLEDGMENTS}

The author would like to acknowledge the technical assistance of F. Robinson and K. Sobnosky. The financial support from NSF CPE-8010868 is also acknowledged. 


\section{REFERENCES}

1. Edwards, V. 1969. Adv. Appl. Microbiol. 11: 157-164.

2. Paul, E. L., A. Kalfman \& W. A. Sklarg. 1981. Ann. N.Y. Acad. Sci. 369: 181-186.

3. Denkewalter, R. G. \& J. Gillin. 1959. Auslegeschrift 1062891.

4. ABbotT, B. S. \& P. Gerhardt. 1970. Auslegeschrift 1062891.

5. TONE, H., A. KitAi \& A. OzaKI. 1968. Biotechnol. Bioeng. 10; 689-692.

6. Kominek, L. A. 1975. Antimicrob. Agents Chemothera. 7: 861-866.

7. Cifgler, A., G. E. N. Nelson \& H. H. Hall. 1962.

8. CYSEWSKi, G. R. \& C. R. Wilke. 1977. Biotechnol. Bioeng. 19: 1125-1130.

9. WANG, H. Y., F. Robinson \& S. S. LeE. 1981. Biotechnol. Bioeng. Symp. 11: 555-560.

10. Spencer, R. R. 1978. Biotechnol. Bioeng. Symp. 8: 257-268.

11. Olmstend, D. R., T. W. Jeffries, R. Naughton \& H. P. Gregor. 1980. Biotechnol. Bioeng. Symp. 10: 247-258.

12. Kominek, L. A. 1975. Antimicrob. Agents Chemother. 7: 856 860 . 